\title{
RESEARCH INTO INFLUENCE FROM DIFFERENT RANGES OF PAR RADIATION ON EFFICIENCY AND BIOCHEMICAL COMPOSITION OF GREEN SALAD FOLIAGE BIOMASS
}

\author{
Leonid B. Prikupets ${ }^{1}$, George V. Boos ${ }^{2}$, Vladislav G. Terekhov ${ }^{1}$, \\ and Ivan G. Tarakanov ${ }^{3}$ \\ ${ }^{1}$ VNISI of S.I. Vavilov, LLC, Moscow; ${ }^{2}$ MPEI NRU, Moscow \\ ${ }^{3}$ RSAU- MTAA (Moscow Timiryazev Agricultural Academy) of K.A. Timiryazev, \\ E-mail:prikup@vnisi.ru
}

\begin{abstract}
Results of the first photobiological studies of optimisation of LED phyto irradiators spectrum and irradiance level, when growing salad-greengrocers plants in greenhouses and plant factories in photoculture conditions, are presented in the article. The results are given as a series of producing capacity curves for salad and basil plants when irradiating by quasi-monochromatic spectrum for three PAR ranges: blue, green and red. In the experiment, levels of photosynthetic photon irradiance $(70 \div 230)$ $\mu \mathrm{mol} / \mathrm{s} \cdot \mathrm{m}^{2}$ and of irradiance $(13 \div 60) \mathrm{W} / \mathrm{m}^{2}$ were varied within a wide range. "Rough" spectra of optical radiation action estimated over producing capacity of plants with different irradiance levels are given, and questions of additivity of different spectral radiation influence in forming vegetable biomass are considered.

Evaluations of efficiency of various PAR intervals for synthesis of biochemical combinations determining nutrition facts of the studied cultures are performed.
\end{abstract}

Keywords: photobiological studies, productivity light curve, photosynthesis, photoculture, LED phytoirradiator (LED PI), photosynthetic active radiation (PAR), action spectrum, photosynthetic photon flux (PPF), photosynthetic photon flux density (PPFD)

\section{INTRODUCTION}

A possibility of creating quasi-monochromatic radiators based on light emitting diodes (LED) for main intervals of PAR range for facilitating biomass formation, morphogenesis and metabolism of plants, stimulates the correspondent photo biological studies around the world. The known $M c$ Cree curve [1], which obtained in vitro using a rather complex optics, describes photosynthesis action spectrum on separate leaves of plants at low levels of irradiance. This curve was a peculiar "Gospel for photo biologists" during about five decades but now it is opened to questions [2]. Due to LEDs, photo biologists have obtained a new effective exploratory tool, which allows carrying out studies of radiation influence of PAR various intervals on producing capacity and quality of plant biomass in a wide interval of irradiance being of interest for the practical photoculture. Such problem namely is set by authors of this work. They have selected as an object of the studies a group of greengrocer vegetable cultures (as exemplified by the Landau salad and the Russian Giant basil, which are of prospective interest for growing in industrial greenhouses).

\section{SHORT CHARACTERISTICS OF THE SPECIFIED CULTURES}

Salads have many versions, and most widespread is leave salad with a rich vitamin and mi- 

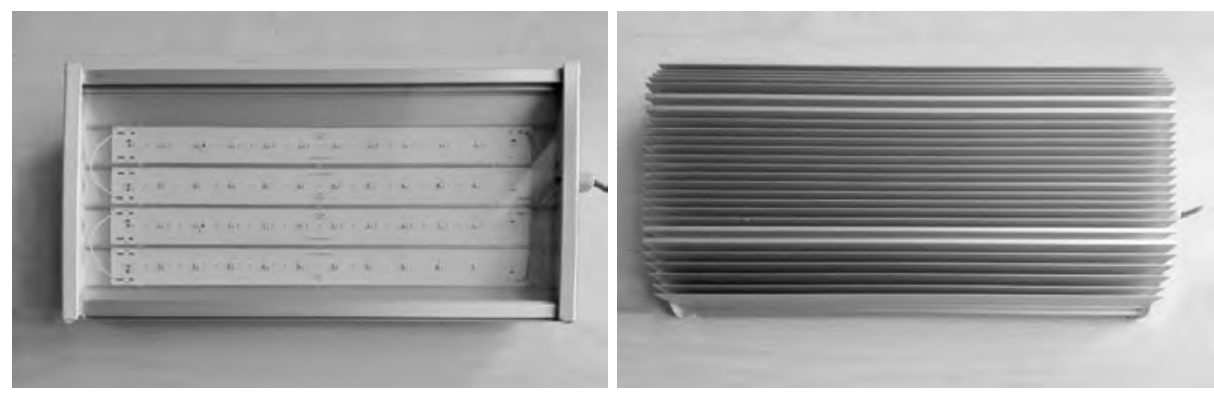

Fig. 1. Appearance of quasi-monochromatic phytoirradiators (PI)

neral composition, including vitamins of B and C groups, as well as calcium, boron, copper, iodine and phosphorus. As a food, salads are very useful for human body.

Basil is a valuable spice-and-flavour culture, and its usefulness for human organism is very big. Most of its necessary for health properties are due to formation of ether oils in its biomass.

\section{RESEARCH METHODS AND EXPERIMENTAL INSTALLATION}

Reaction of plants on influence of quasi-monochromatic radiation of some PAR intervals when changing within a wide range of irradiance was investigated. The reaction of plants means change of gross weight of economically-useful biomass (producing capacity). In the specified conditions, concentration of most important components of the product biochemical composition determining its nutrition value was estimated.

As a result of the studies, it was supposed to obtain for each spectral version a series of productivity light curves, based on which a real efficiency of separate PAR ranges can be estimated, and search of phytoirradiators (PI) spectrum and irradi-
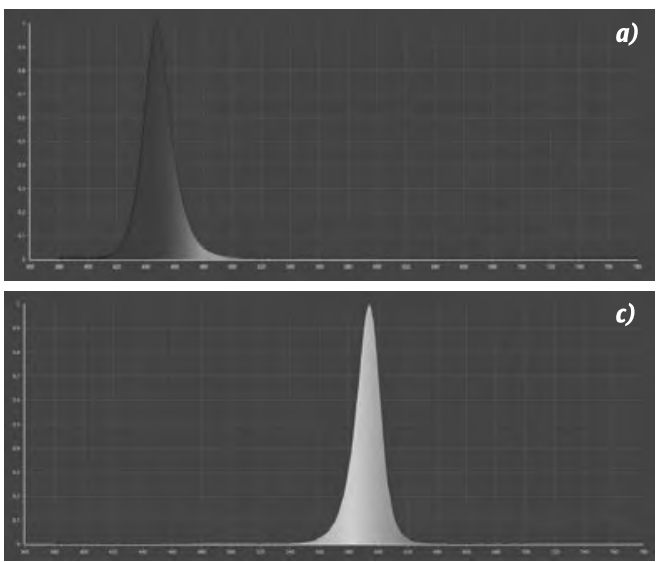

ance optimum requirements can be determined for plants growing in accordance with the photoculture technology.

Especially for these studies, in LLC International Lighting Corporation BOOS LIGHTING GROUP and in the VNISI, a series of quasi-monochromatic PIs was developed and manufactured. Their parameters are presented in Table 1. Fig. 1 shows photos of PIs, in which colour selective LEDs of Cree Company (USA) were used.

Spectra of optical radiation of the quasi-monochromatic PIs used in the research are presented in Fig. 2.

It can be easy seen from Fig. 3 that "blue", "green" and "red" PI radiation is in the sensitivity areas of most pigments being photodetectors of plants.

In the PIs, controlled drivers of OT180W/ UNV1250C type were used. Current adjustment within $(350 \div 1300)$ mA allowed changing power of irradiators and irradiance over the technological area.

To estimate a possible interval of irradiance control, dependence of radiation flux relative value on current was previously measured for each PI type (Fig. 4).

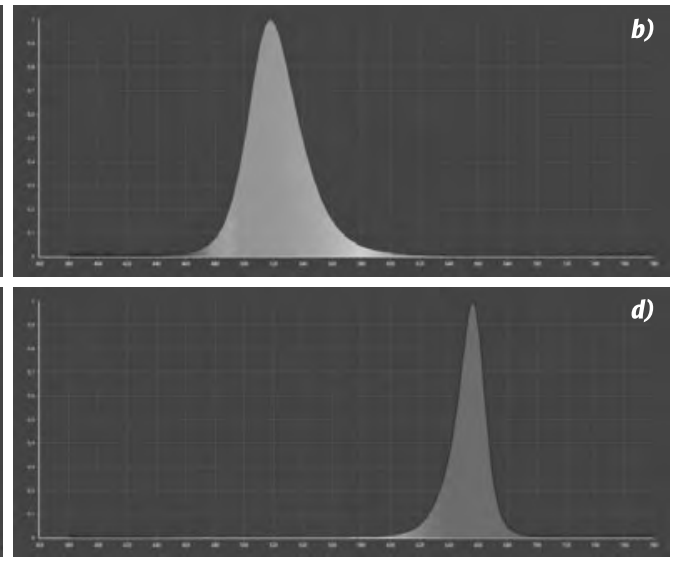

Fig. 2. Spectra of radiation quasi-monochromatic PI used in the research: (a) blue, (b) green, (c) amber, (d) red 


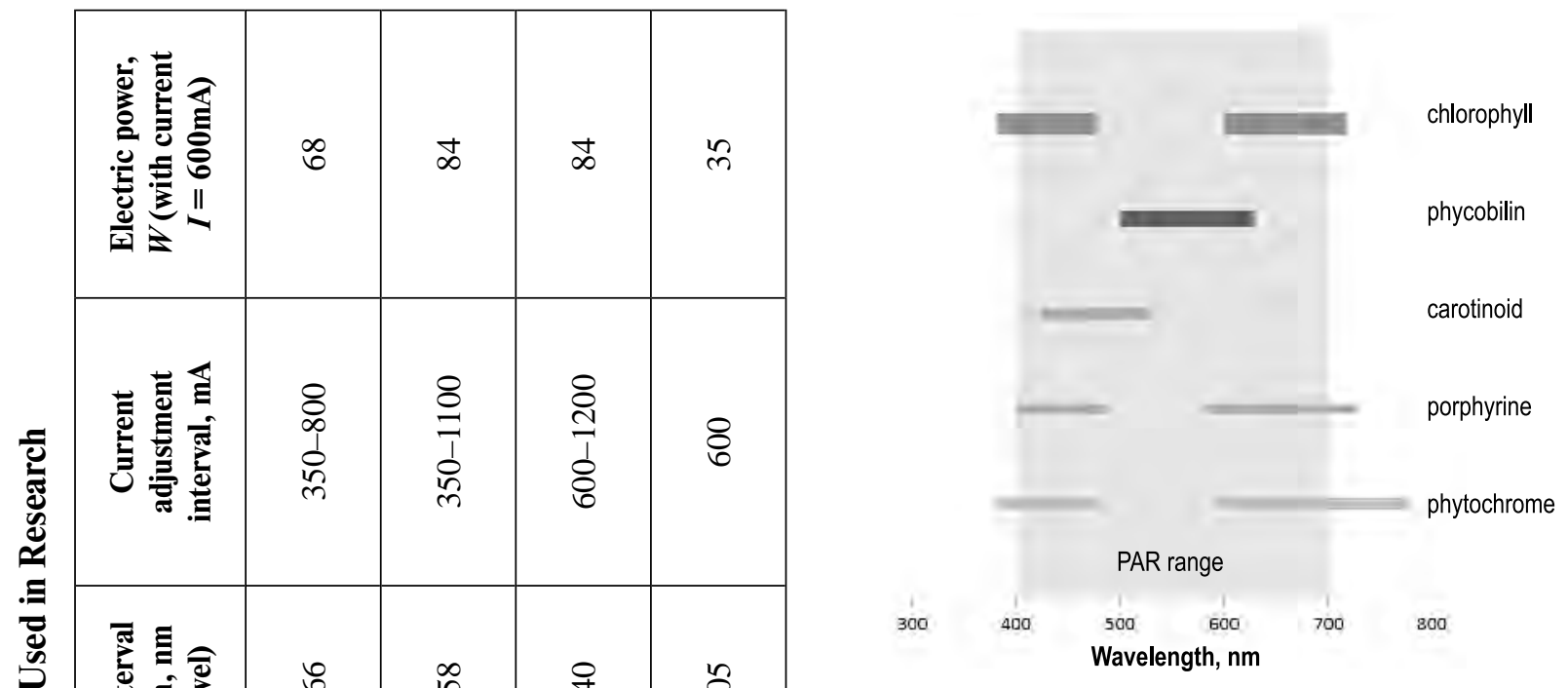

Fig. 3. Spectral intervals of effective absorption of main plant photopigments

The studies were carried out using a phytothrone of the Artificial Climate Laboratory in the RSAUMSHA of K.A. Timiryazev on an exploratory complex consisting of six unified modular installations with reflecting screens of specula film. General view of the installations is given in Fig. $5^{1}$. The interval of possible changes of irradiance in the LED modules was set within $(60 \div 300) \mu \mathrm{mol} / \mathrm{s} \cdot \mathrm{m}^{2}$. In order to obtain the data necessary to create productivity light curves with stable irradiance values for a specific spectral version, not less than four vegetations were performed. Choice of the working area (size of placing plants on the bedding area) was determined by the requirement to provide irradiance uni-

1 In parallel with the study of exposure of quasi-monochromatic radiation fluxes, experiments with radiation sources of a continuous spectrum in the PAR interval were made. The results will be presented in a separate article.

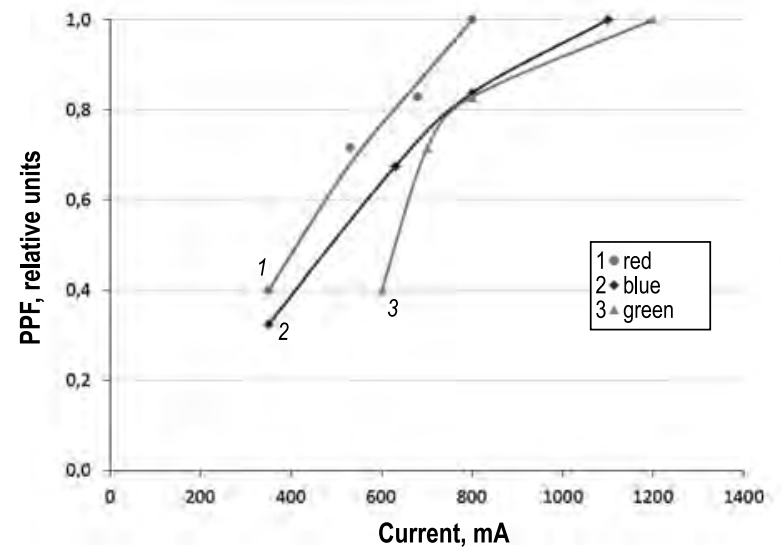

Fig. 4. Photosynthetic photon flux current dependence for Phytoirradiators 

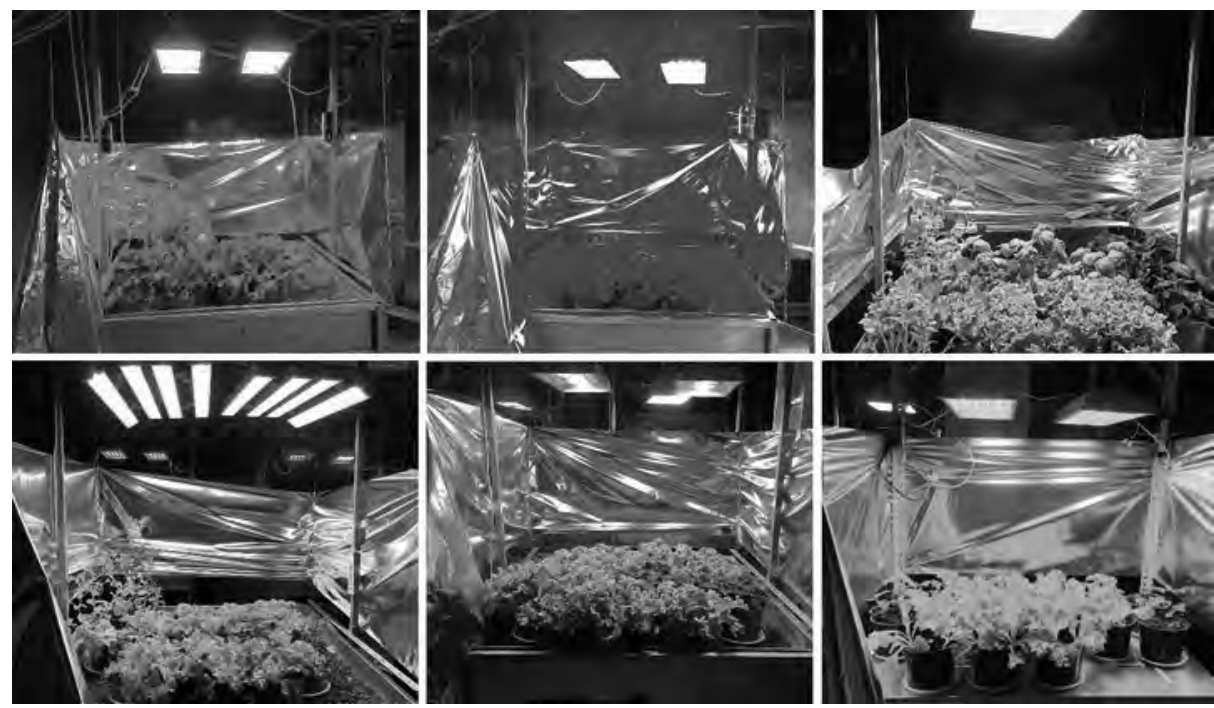

Fig. 5. General view of the research installations

formity $E_{a v}= \pm 10 \%$. The working area was equal to $(0.25 \div 0.35) \mathrm{m}^{2}$ depending on the conditions of a specific experiment. Adjustment of the irradiance was made according to the flow chart in Fig. 6 by means of a programmer and a computer with specially installed software. To determine spectrum of PI radiation, photosynthetic photon and energy irradiance in the PAR interval, the $\mathrm{Li}-250 \mathrm{~A}$ device for PPFD integral measurements with quantum detector Li-190R (Li-COR Company, USA) and MK 350S spectrometer (UPRtek Company, Taiwan) were used. At the request of the authors, INTEX Lighting Company (St. Petersburg) by agreement with UPRtek Company installed an additional option on the MK 350 S device, which allows measuring irradiance within the PAR interval using energy units $\left(\mathrm{W} / \mathrm{m}^{2}\right)$. Choice of the devices was an extraordinary problem, because it was connected with introduction of GOST P 57671-2017 and PNST 2112017 developed in the VNISI [3-5].

Besides the lighting parameters of the studies methods stated above, other experimental conditions corresponded to the requirements of the technology of growing salad-greengrocers cultures accepted in the phytothrone are briefly described below.

The experiments were made during a constant photo period of $18 \mathrm{~h}$. Air temperature in the day-time was $22^{\circ} \mathrm{C}$, and at night $18^{\circ} \mathrm{C}$. The plants were grown up in vegetative containers with the "Agrobalt C" nutritious mixture by tree pieces of salad, or five pieces of basil. As a substratum, an upper neutralised sphagnous peat of a low decomposition level with humidity no more than $65 \%$ was used. It contained calciferous (dolomitic) powder, combined fertilizer $\left(\mathrm{N}_{\text {gen }}=150 \mathrm{mg} / \mathrm{l}, \mathrm{P}_{2} \mathrm{O}_{5}=150 \mathrm{mg} / \mathrm{l}, \mathrm{K}_{2} \mathrm{O}=250 \mathrm{mg} / \mathrm{l}\right.$, $\mathrm{Mg}=30 \mathrm{mg} / \mathrm{l}, \mathrm{Ca}=120 \mathrm{mg} / \mathrm{l}$ and microelements). Its $\mathrm{pH}$ was equal to 5.5-6.6. Watering was carried out by mass with quadruple frequency. The humidity of full moisture capacity was maintained to be equal to $70 \%$.

Account of the harvest biomass and other determinations were performed in 35 days beginning from the seedling, general biomass for every container was also taken into consideration.

Concentration of vitamin C (ascorbic acid) in plant tissues was determined by a method based on Tillmans's reaction with decolouration of 2.6-dichlorophenol-indophenol. Nitrate concentration was determined using an ion-selective electrode after extraction with aluminium potassium sulphate. To determine quantitative and qualitative composition of accumulated biologically active aromatic components being a part of basil ether oil, gas chro-

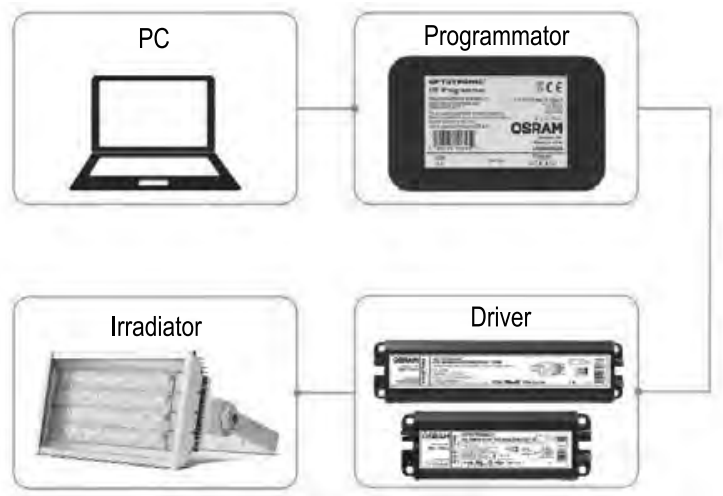

Fig. 6. Flow chart for power and PPF of quasi-monochromatic PI adjustment 

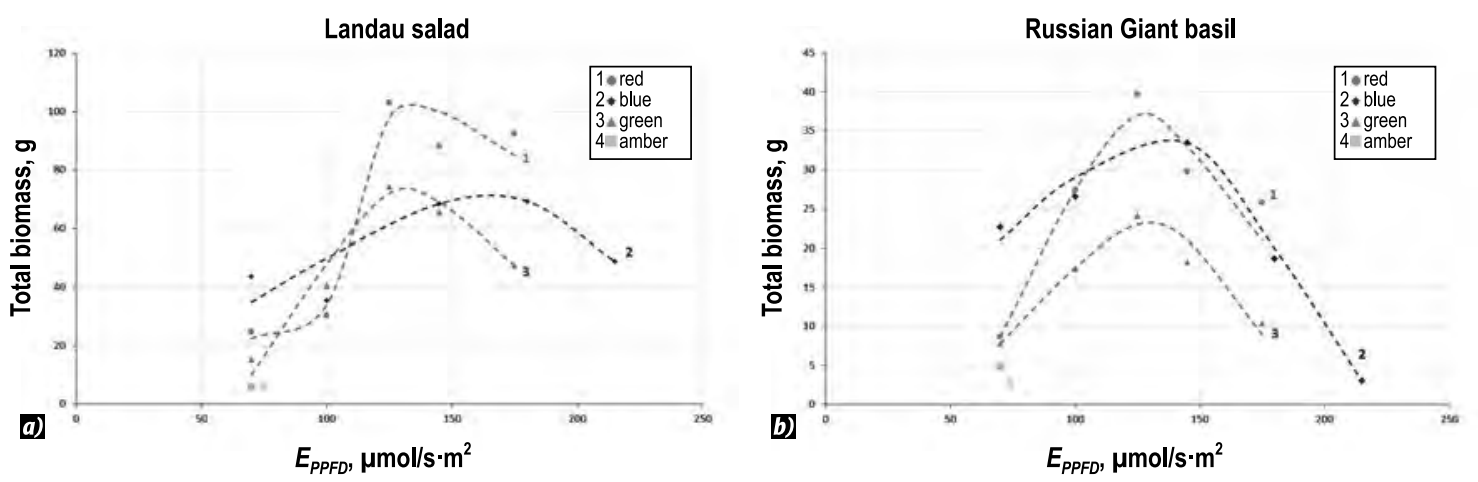

Fig. 7. A series of productivity light curves of salad (a) and basil (b) for the photosynthetic photon values
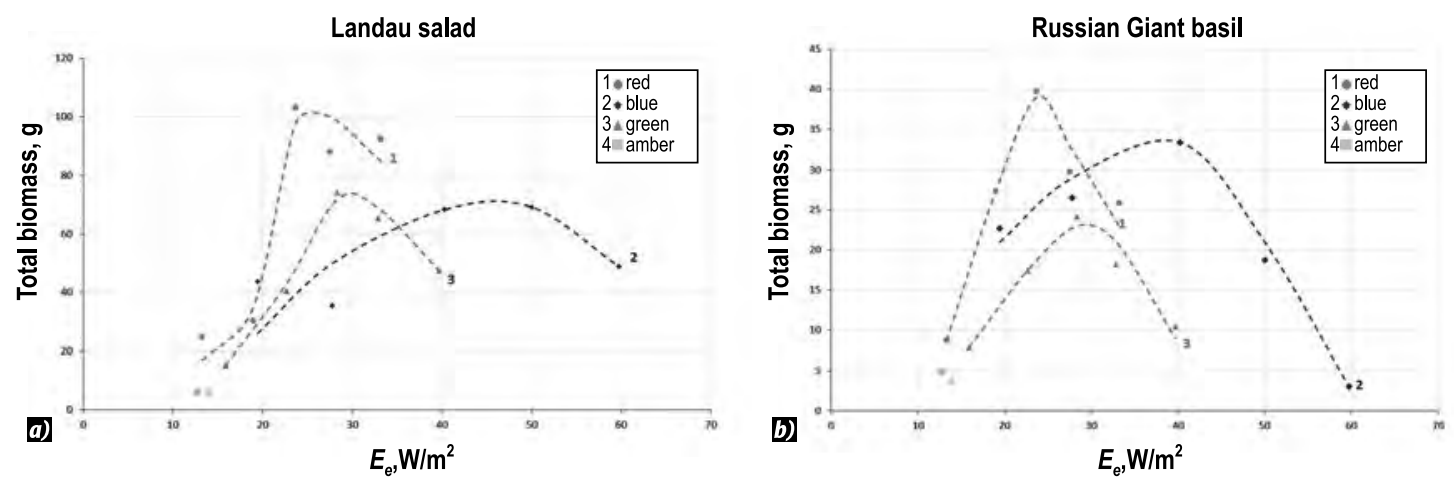

Fig. 8. A series of productivity light curves for plants of salad (a) and of basil (b) for the energy values

matography was used with mass-selective flamingly-ionisation detection. Identification of the ether oil components was carried out using mass-spectra libraries.

\section{ANALYSIS OF THE RESEARCH RESULTS}

At present, the fact is practically assured that photosynthesis is a quantum process, in which quantum efficiency (or intensity of photosynthesis) depends on the absorbed photon number within the PAR interval. Thereupon, photosynthetic photon values are now actively introduced in practice of radiation metrics in protected ground constructions, primarily in greenhouses with the plant photoculture.

It should be remembered, however, that photosynthesis in a real plant being a photobiological system has in essence stochastic nature. It is shown in theoretical photobiology, from 8 to 12 photons are corresponding to produce one molecule of the organic substance or $\mathrm{O}_{2}$ [6]. It follows from this that even for photosynthesis, without mentioning vegetable biomass process synthesis, the photon values is no more than a convenient measure for conventional character calculations.
In connection with the specified above and taking into account the photonic metrological opportunities, it is advisable not to abandon the energy values for the PAR interval very popular in the last century in the photobiological studies and even in the plant practical photoculture ${ }^{2}$. An integrated energy "thread" is rather distinctly traced. This thread connects the consumed electric power of a lamp, part of its radiation in the PAR interval, efficiency of a luminaire, PAR usage coefficient in an illumination device (ID) from the one hand, with the technological area irradiance, the ID specific power consumption and even with the energy equivalent of the produced biomass from the other hand.

From these point of view, it is especially interesting, based on the research data, to consider the results of biomass synthesis being the function of both photon and energy irradiances, a connection between which is determined by the following expression:

$$
E_{p h}=K_{e p h} \cdot E_{e}
$$

2 A preference to the energy values is given by some leading plant photo-physiologists, in particular by professor. A.A. Tikhomirov [7]. 

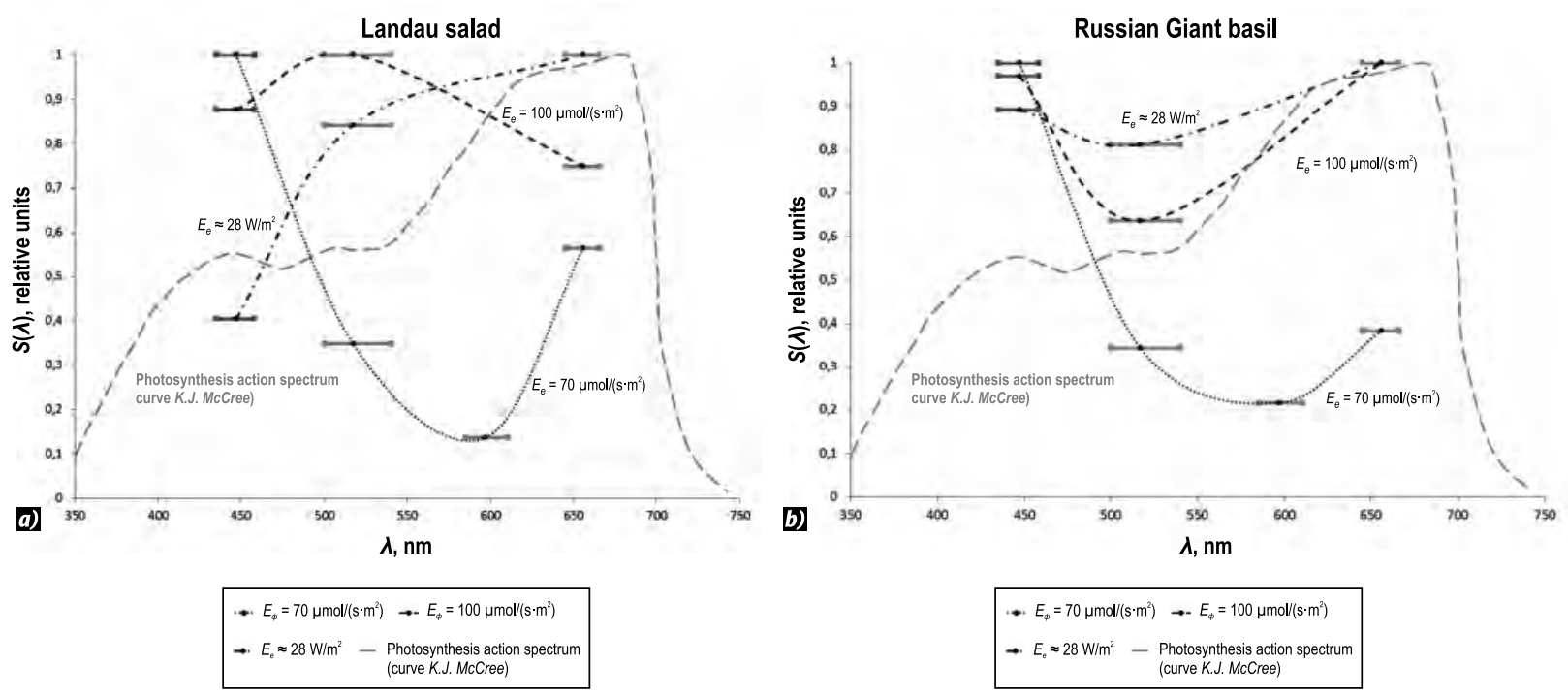

Fig. 9. "Rough” action spectra of salad biomass (a) and of basil (b) synthesis

where

$$
K_{e p h}=\frac{\frac{1}{h \cdot c \cdot N_{A}} \cdot \int_{400}^{700} e(\lambda) \cdot \lambda d \lambda}{\int_{400}^{700} e(\lambda) \cdot d \lambda},\left[\frac{\mu \mathrm{mol}}{J}\right],
$$

where $e(\lambda)$ is the spectral concentration of irradiance, $\left[\mathrm{W} \cdot \mathrm{m}^{-2} \cdot \mathrm{nm}^{-1}\right], \lambda$ is the radiation wavelength in the PAR interval, [nm], $h$ is the Plank constant, [J·s], $c$ is the light velocity, [m/s], $N_{A}$ is the Avogadro constant, $\left[\mu \mathrm{mol}^{-1}\right]$.

A transition from $E_{e}$ to $E_{p h}$ or vice versa is possible by means of a calculation or using the correspondent type devices, which, as it was stated above, were used in this work.

The research main results presented as producing capacity light curves for salad and basil plants are given in Figs. 7-8.

For the presented producing capacity average values, root-mean-square deviation was equal to \pm $10.3 \%$ (salad) and $\pm 18.5 \%$ (basil).

It should be noticed that growing of plants under quasi-monochromatic radiation, i.e. in the light mode, when throughout all vegetation a part of photosynthesis spectrum only is involved, allows not only obtaining direct data on efficiency of a certain PAR interval but also estimating possibilities of a plant as self-controlled system.

An analysis of Landau salad producing capacity dependence on the PPFD level shows a rather abrupt plant reaction to low-energy red radiation quanta. With low irradiance, the reaction efficiency was lower than for blue radiation. With average PPFD equal to $100 \mu \mathrm{mol} / \mathrm{s} \cdot \mathrm{m}^{2}$, the effect was slightly lower than for blue radiation and even for green, but within the higher irradiance interval of about $(130 \div$ 140) $\mu \mathrm{mol} / \mathrm{s} \cdot \mathrm{m}^{2}$, a maximum level of producing capacity for all series of the experiences was reached.

Length of the "ascending section" of the productivity light curve is the biggest for blue radiation. Maximum values of producing capacity are reached in this case at the PPFD about $(170 \div 180) \mu \mathrm{mol} /$ $\mathrm{s} \cdot \mathrm{m}^{2}$. This fact confirms the previously obtained data [8] and means that support of high part of blue spectrum irradiation in LED phytoirradiators is not prospective for industrial growing of salad cultures.

The results of this research demonstrate that green interval of the spectrum when growing salad is not an "outlaw" at all and provides a higher producing capacity than red and blue radiations at PPFD equal to $100 \mu \mathrm{mol} / \mathrm{s} \cdot \mathrm{m}^{2}$.

An experiment with a specially made yellow-orange (amber) PI with $\mathrm{E}=70 \mu \mathrm{mol} / \mathrm{s} \cdot \mathrm{m}^{2}$ is a direct confirmation of the assumption of a low efficiency of the yellow-orange PAR interval, which radiation is absorbed practically by carotinoids and phycobilins only. In further experiments, this LED irradiator was not used.

As it was stated above, when transiting from PPFD scale to energy irradiance $E$ scale, $\mathrm{W} / \mathrm{m}^{2}$ (Fig. 8a), in the results data for the first time, we could notice an addition influence of PAR various intervals on producing capacity of the salad due to the new hardware abilities. Naturally, the scale inversion slightly changed relative position of the light curves, significantly expanded the experiment 

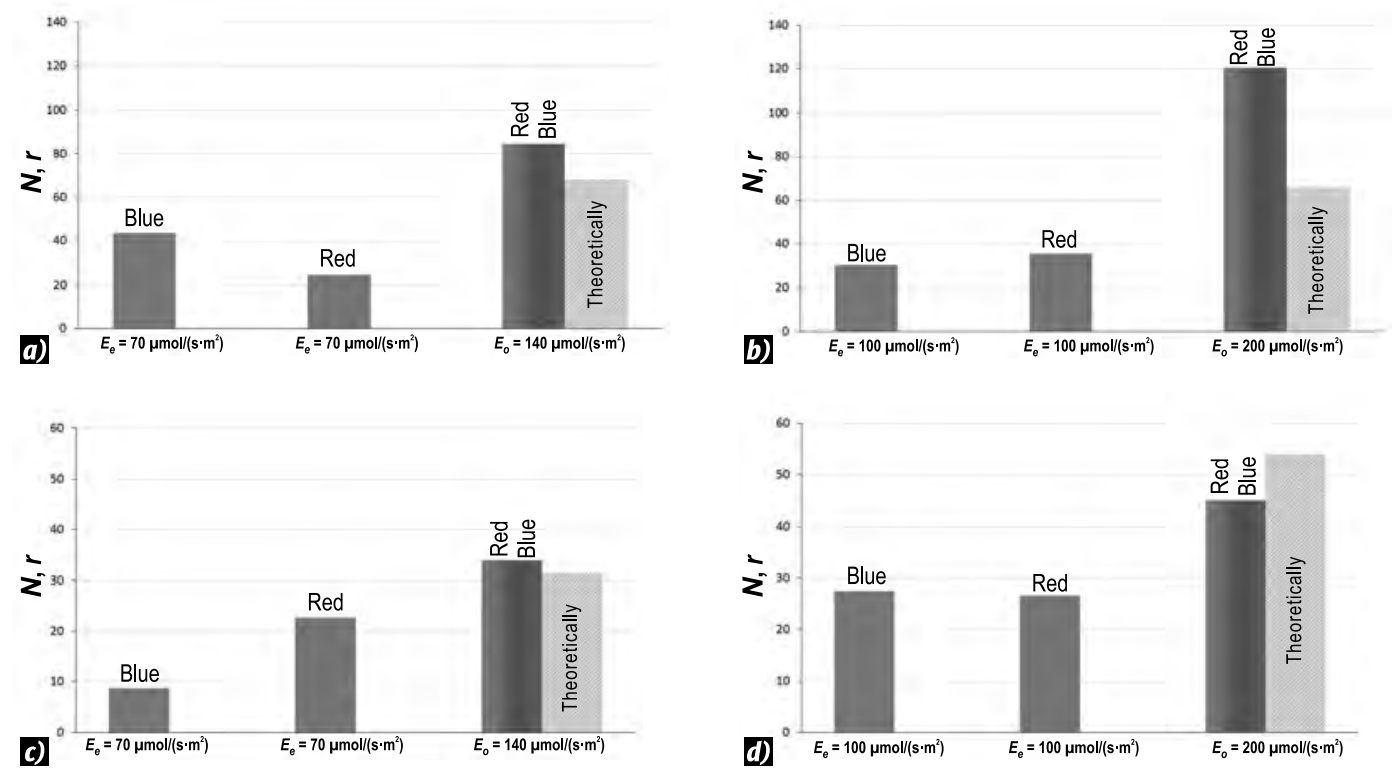

Fig. 10. Additivity of action of different-spectral PAR range in useful biomass synthesis $\mathrm{N}$

range and presented the data in the form more habitual for lighting engineers and photobiologists.

It should be noticed that the productivity light curve for blue PAR interval for the energy value has the most flat nature and reaches a producing capacity maximum with rather high irradiance $(40 \div 50)$ $\mathrm{W} / \mathrm{m}^{2}$, which is considerably lower than for red radiation optimum with $E$ in range $(23 \div 25) \mathrm{W} / \mathrm{m}^{2}$.

Productivity light curves for the Russian giant basil somewhat differ from the salad curves. PAR red interval is also the most effective to provide a high producing capacity. An experiment with red radiation at $E>140 \mu \mathrm{mol} / \mathrm{s} \cdot \mathrm{m}^{2}$ revealed an effect of a quick decreasing plant growth, which is hard to be explained. In the curve family $N=f(P P F D)$, positions of maxima for all PAR intervals are very close and correspond to $E$ about $(125 \div 150) \mu \mathrm{mol} / \mathrm{s} \cdot \mathrm{m}^{2}$.

Apparently for industrial basil growing, an optimum by spectrum will be a combination of red and blue PAR intervals, because green radiation efficiency is significantly lower.

Inversion of the irradiance scales for basil leads to a noticeable change of the productivity light curve positions probably connected with different energy "weights" of green, red and blue photons (Fig. 8b.).

Maxima of dependences in the irradiance scale of red and blue PAR intervals are displaced by $\Delta E \approx 15 \frac{\mathrm{W}}{\mathrm{m}^{2}}$, which indisputably provides essential energy efficiency advantages for red radiation.
The data under consideration are in addition vividly illustrated using so-called "rough" action spectra of biomass synthesis of the two studied cultures $^{3}$ (Fig. 9). The action spectrum is a reaction of plants (in this case it is producing capacity) to radiation within separate spectral intervals with a constant irradiance (photosynthetic, photon or energy). The action spectrum allows determining influence of a certain photopigment type on biomass synthesis.

As it follows from the data (Fig. 9) the producing capacity action spectrum character drastically depends on the irradiance $\left(\mu \mathrm{mol} / \mathrm{s} \cdot \mathrm{m}^{2}\right.$ or $\left.\mathrm{W} / \mathrm{m}^{2}\right)$. Depending on the producing capacity value, efficiency of a specific PAR interval can differ radically. From here, an uncertainty of many research results, which were carried out for one or two irradiance levels only with contradictory data, is quite understandable, and this is discussed, for example, in [10].

Introducing the methodological content only into our research next element and considering it as a prologue to the following experiments, we made some tests to estimate an additivity of radiation influence of blue and red PAR intervals on biomass synthesis.

Generally the additivity is a property of values, which is consists in the fact that a va-

3 The concept of a "rough" spectrum is introduced in [9] and used, when width of the selected spectral interval is equal to several tens nanometres. 


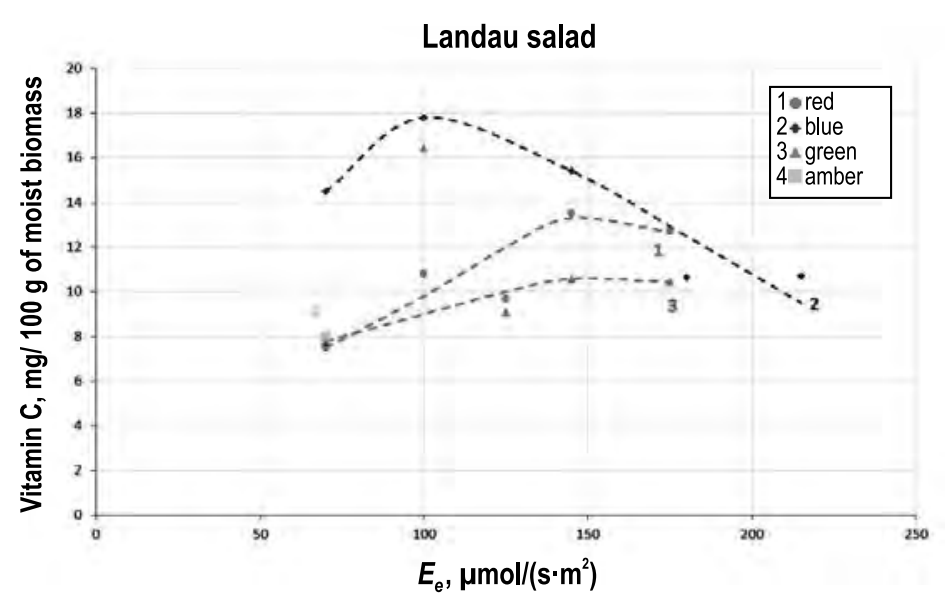

Fig. 11. Dependence of vitamin C concentration for Landau salad on the PPFD level

lue relating to the whole volume and correspondent to it, is equal to the sum of the values corresponding to its parts. So any arbitrary function $f$ is additive, if

$$
f\left(x_{1}+x_{2}\right)=f\left(x_{1}\right)+f\left(x_{2}\right)
$$

If the specified equality is not observed, and relation of the left and right parts of equation (2) $f\left(x_{1}+x_{2}\right) /\left(f\left(x_{1}\right)+f\left(x_{2}\right)\right)>1$, then the considered function can be named superadditive, and if this relation is $<1$, then the function is subadditive.

It should be noticed that $\mathrm{McCree}$ in his experiments with photosynthesis of an insulated leaf doubted that different-spectral radiation influence is additive [1]. According to (2), the additivity criterion can be formally written down as follows:

$$
A=\frac{N\left(E_{0}\right)}{\sum_{i=1}^{n} N_{i}},
$$

where $E_{0}=\sum_{i=1}^{n} E_{\Delta \lambda_{i}}, N\left(E_{0}\right)$ is the producing capacity in case of combined action of $n$-spectral intervals with general irradiance $E_{0}, N_{i}$ is the producing ca-

pacity of quasi-monochromatic irradiator $i$ radiating in the wavelength interval $\Delta \lambda_{i}$ and providing photon irradiance $E_{\Delta \lambda_{i}}$.

As it was stated above, in our research, $n=2$, and for each experience, $E_{\Delta \lambda_{i}}=$ const .
When carrying out the additivity studies, it is expedient to select $E_{\Delta \lambda_{i}}$ levels on the ascending sec-

tions of the correspondent productivity light curves. For these reasons, in our experiment PPFD were selected as $E_{\Delta \lambda 1}=70 \mu \mathrm{mol} / \mathrm{s} \cdot \mathrm{m}^{2}$ and $E_{\Delta \lambda_{2}}=$ $100 \mu \mathrm{mol} / \mathrm{s} \cdot \mathrm{m}^{2}$. Thus for $E_{\Delta \lambda i}$ according to (2), two values $140 \mu \mathrm{mol} / \mathrm{s} \cdot \mathrm{m}^{2}$ and $200 \mu \mathrm{mol} / \mathrm{s} \cdot \mathrm{m}^{2}$ were set.

The results for salad and basil plants presented in Fig. 10 are quite logical and explainable. For the primary level of $E_{\Delta \lambda_{i}}=70 \mu \mathrm{mol} / \mathrm{s} \cdot \mathrm{m}^{2}$, the obtained

effect for both cultures can be estimated as superadditive (according to (3), A>1). This confirms perspective of further research by optimization of binary red-blue PI spectrum. A qualitatively similar result is also obtained for salad plants with a higher primary PPFD $\mu \mathrm{mol} / \mathrm{s} \cdot \mathrm{m}^{2}$ taking into account the productivity light curve flat nature at high PPFD levels. The subadditivity effect of red-blue radiation at $E_{\Delta \lambda_{i}}=100 \mu \mathrm{mol} / \mathrm{s} \cdot \mathrm{m}^{2}$ for basil can be ex-

plained by a more abrupt nature of the productivity light curve decrease already with PPFD higher than $150 \mu \mathrm{mol} / \mathrm{s} \cdot \mathrm{m}^{2}$.

The fact of influence of PAR on plant synthesis for some biochemical compositions determining nutrition value of vegetable products [11] is well known. According to the modern concepts, much attention is paid to this question when growing products of an increased ecological quality under the photoculture conditions.

At this stage of research, we wanted to obtain estimation data assuming then to clarify them 
and to lead to a level of technological indicators at the closing stage of the studies.

As the main component of the biochemical composition for Landau salad, vitamin C concentration of 1mg/100 g of biomass was accepted, and for basil plants it was concentration of eugenol being the main type of ether oil. Concentration of nitrates in biomass was also estimated.

Fig. 11 shows a series of dependences on vitamin $C$ concentration in moist biomass of Landau salad. A general effect for all three PAR intervals is an increase of ascorbic acid concentration with increase of irradiance. And position of maximum for the blue interval corresponds to lower irradiance than for the producing capacity light curve. In the PPFD interval, which is of interest for practical photoculture, concentration of vitamin $\mathrm{C}$ can be $(20 \div$ $40) \%$ higher than for salad cultures average level (10 mg/100 g).

During the studies of the spectrum and of the PAR irradiance exposure on biosynthesis of eugenol in basil, one could not reveal a stable influence of them. High share eugenol concentration rates $(50 \div$ $55) \%$ were stably revealed when irradiating with red light of PPFD $(125 \div 150) \mu \mathrm{mol} / \mathrm{s} \cdot \mathrm{m}^{2}$.

By nitrate concentration, parameter dispersion was at the first order values level while remaining $(2 \div 3)$ times lower than the maximum allowable value (2500 mg/kg).

\section{CONCLUSION}

The photobiological research was performed using the last achievements of light engineering are indisputably important and show great opportunities of LED radiators for development of reasoned requirements to systems of phytoillumination for plant photoculture of different types.

For two types of vegetative plants in a wide intervals of PPFD $\left[\mu \mathrm{mol} / \mathrm{s} \mathrm{m}^{2}\right]$ and irradiance [W/ $\mathrm{m}^{2}$ ], data of the main spectral PAR intervals influence on producing capacity is obtained, and maximum efficiency of the PAR red interval is confirmed.

The research results given in the article firmly show that reaction of plants (producing capacity) not additively changes depending on spectral composition and irradiance, and that searches of universal spectra influencing plant producing capacity are doubtful. A producing capacity action spectrum even for vegetative plants depends on the irradiance and should be in general estima- ted using two-dimensional scales ( $\lambda$ and $E$ ). Summarising the all above said, it should be noticed that at present there is no alternative for the experimental method of optimising key lighting parameters for plant photoculture.

Along with the scientific and practical importance, studies of photophysiological efficiency of PAR separate intervals have methodical nature and can be applied in training courses for students of the following specialties: "Light and Engineering" and "Physiology of Plants".

\section{ACKNOWLEDGMENT}

This article is written within a project of financial support of applied scientific researches by the Ministry of Education and Science of the Russian Federation. The subject is: "Comprehensive studies in the plant photoculture and creation of high-effective LED phytoirradiators for increase of energy efficiency of industrial greenhouses"

Agreement on granting subsidy is the \#14.576.21.0099 of 9/26/2017. Unique identifier: RFMEFI57617X0099.

\section{REFERENCES}

1. McCree K.J. The action spectrum, absorbance and quantum yield of photosynthesis in crop plants // Agric. Meteorology, 1972, pp. 192-216.

2. http://www.hortibiz.com/item/news/research-redefining-the-mccree-curve/ 26.04.2018.

3. Prikupets L.B. Technological Lighting for Agro-Industrial Installation in Russia // Light \& Engineering, 2018, Vol.26, № 4, pp. 7-17.

4. Boos G.V., Prikupets L.B., Rozovsky E.I., Stolyarevskaya R.I. Standardization of Lighting Fixtures and Installation for Greenhouses // Light \& Engineering, 2017, Vol.25, № 4, pp. 18-24.

5. Bartsev, A., Prikupets, L., Shakhparunyants, A. Measurements of photosynthetic photon flux (PPF) and flux density (PPFD) for greenhouse LED irradiators // PROCEEDINGS of CIE2018 “Topical Conference on Smart Lighting”, 2018, pp.564-569.

6. Konev S.V., Volotovsky I.D. Photobiology // Minsk: BSU publishing house of V.I. Lenin, 1979, $384 \mathrm{p}$.

7. Tikhomirov A.A., Sharupich V.P., Lisovsky G.M. Photoculture of plants // Novosibirsk: Publishing house of the Siberian Branch of the Russian Academy of Sciences, 2000, $202 \mathrm{p}$. 
8. Tikhomirov A.A., Lisovsky G.M., Sidko F. Ya. Light spectral composition and producing capacity of plants / /Novosibirsk: Nauka, 1991, 168p.

9. Tokhver A.K. Phytochrome, its main configurations and their properties // In the collection: Photoregulation of metabolism and morphogenesis of plants, Nauka, 1975, pp. 56-65.

10. Bugbee B. Towards optimal spectral quality for plant growth and development: The importance of radiation capture // Plants, Soils, and Climate Faculty Pub- lications, 2016. URL: https://digitalcommons.usu.edu/ cgi/viewcontent.cgi?article $=1765 \&$ context=psc_fac-pub (31.08.2018).

11. Berkovich Yu.A., Konovalova I.O., Smolyanina S.O., Erokhin A.N., Avercheva O.V., Bassarskaya E.M., Kochetova G.V., Zhigalova T.V., Yakovleva O.S., Tarakanov I.G. LED crop illumination inside space greenhouses // REACH - Reviews in Human Space Exploration., 2017, Vol. 6., pp. 11-24.
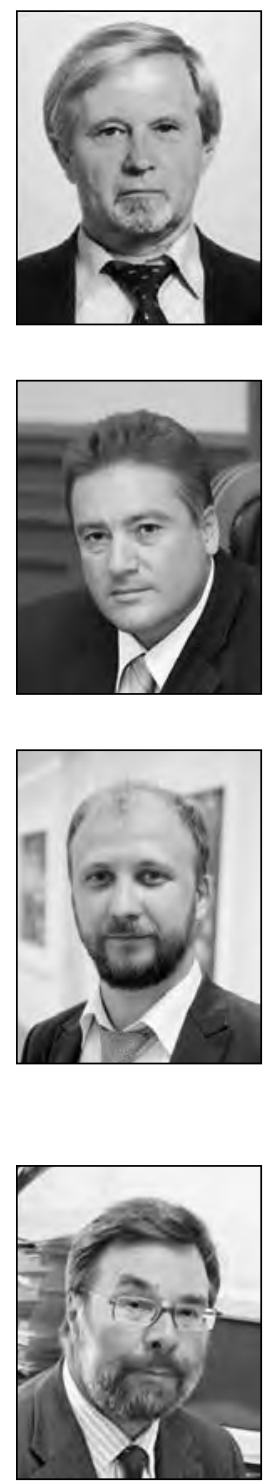

\section{Leonid B. Prikupets,}

Ph.D. Graduated with honors from MPEI in 1970. At present, he is the head of laboratory in VNISI by S.I. Vavilov and member of editorial board of Svetotekhnika and Light \& Engineering Journals

\section{George V. Boos,}

Ph.D., graduated from MPEI in 1986, President and Member of Board of Directors of BL Group, Head of the chair "Light and Engineering” NIU MPEI. He is a Laureate of the State Prize of the Russian Federation (for architectural illumination of Moscow), chairman of the editorial board of the "Svetotekhnika" and "Light \& Engineering” Journals, Member of the Russian Academy of Natural Sciences

\section{Vladislav G. Terekhov,}

engineer. He graduated with honors from Moscow State University of Economics, Statistics and Informatics with a degree in Applied Informatics in Economics, and at present, he is a Head of the Department for the Promotion of LLC International Lighting Corporation BL GROUP and researcher in VNISI of S.I. Vavilov. He is a member of the Organizing Committee of the All-Russian Conference "Gallium, Indium and Aluminum Nitrides. Structures and devices" and an expert on energysaving technologies in the lighting of the Russian Union of Builders

\section{Ivan G. Tarakanov,}

Prof., Dr. of Biological Sc. He graduated in 1978 from the Moscow Agricultural Academy of K.A. Timiryazev. At present, he is a Head of Department "Plant Physiology” RGAU-MTAA of K.A. Timiryazev 